\title{
Correlation Studies of Paint Adhesion to Surface Morphology of Compatibilized PPE / PA Blends
}

\author{
Nisha Preschilla*, A.V.S. Kishore ${ }^{*}$, Toob Meerman ${ }^{* *}$, Roshan Jha* \\ *GE India Technology Center, Hoodi Village, Whitefield Road, Bangalore-560066, India \\ ${ }^{* *}$ GE Advanced Materials-Europe, Plasticlaan-1, Bergen op Zoom, The Netherlands
}

Compatibilized blends of Polyamide (PA) and Polyphenylene ether (PPE) have been used extensively for automotive exterior body panels due their combination of physical properties, chemical resistance and processability [1]. An important attribute of these materials is their ability to be painted with typical high gloss automotive paint systems. While surface properties such as morphology, composition, PA crystallinity etc. might be expected to influence paint adhesion, a systematic study towards identifying the possible factors that enhance or deteriorate paint adhesion has not been reported.

This report briefly describes surface characterization studies of various factors believed to influence paint adhesion performance of compatibilized, impact modified PPE / PA blends. It has been observed that for specific paint systems, painting processes and the standard steam jet adhesion test method [2] formulated by the automotive manufacturers, paint adhesion varies with changing molding conditions. Detailed surface characterization of selected samples - with paint adhesion ranging from extremely good to poor - was carried out with transmission electron microscopy (TEM), X-ray photoelectron spectroscopy (XPS) and X-ray diffraction (XRD). TEM studies suggest that surface morphology; the size and shape of PPE domains in particular, play a vital role in paint adhesion performance. A non-sheared surface morphology, with uniformly dispersed PPE domains having a size distribution centered about $1.5 \mu \mathrm{m}$ (Fig. 1a), was consistently observed to result in painted specimens possessing good paint adhesion. The presence of sheared PPE (Fig. 1b), densely dispersed small PPE domains with an average size distribution less than $0.5 \mu \mathrm{m}$ (Fig. 1c), or clustered PPE domains (Fig. 1d) at the surface were observed in painted specimens possessing poorer paint adhesion. In the case of samples with highly sheared PPE domains (Fig. 1b), stress relaxation leading to initiation of micro-cracks at the surface may explain poor paint adhesion. All three morphologies shown in Fig. 1 (b) - (d) appear to have lesser availability of PA on the surface. PA as a polar material is believed to play an important role in obtaining good paint adhesion. XPS analysis of these samples supports this (Table 1) as seen by the differences in surface PA content; $73 \%$ for the samples with good adhesion as compared to PA content as low as about $57 \%$ for samples with poor paint adhesion. XRD studies (Fig. 2) suggest that PA crystallinity did not significantly differ in samples having good or poor paint adhesion. From these experiments, surface morphology would appear to play a key role in paint adhesion in compatibilized PPE / PA blends. This suggests that optimization of molding parameters to obtain the desired morphology rich in surface PA content may directly enhance paint adhesion performance of a molded body panel.

\section{References}

[1] C. Chiang, F. Chang, Polymer, 38, 19, 4807, 1997

[2] K. Jaehnichen, J. Frank, D. Pleul, F. Simon, J. Adhesion Sc. Tech., 17 (12): 1635-1654, 2003 

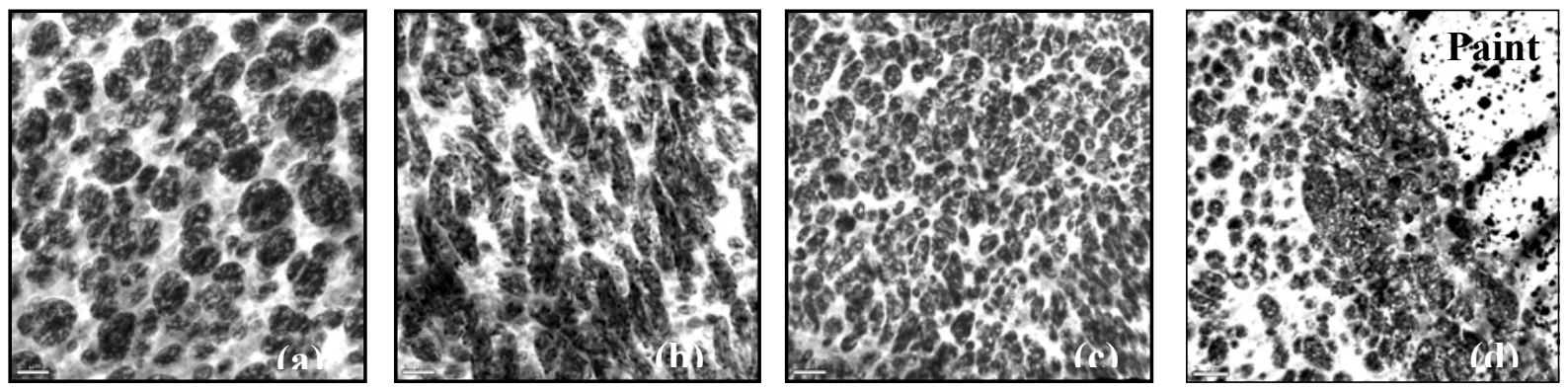

Fig. 1. Surface morphology of PPE / PA blends, the continuous matrix phase is PA, while the dispersed phase is PPE. (a) Typical surface morphology of a sample with good paint adhesion; well dispersed PPE domains with particle size distribution $1.5 \pm 0.2 \mu \mathrm{m}$ is seen. (b) - (d) shows surface morphology of samples with poor paint adhesion indicating that sheared / clustered PPE or very small PPE particle size distribution adversely affect paint adhesion. Scale bar $=1 \mu \mathrm{m}$.

Table 1. XPS data showing surface composition of samples with good and poor paint adhesion

\begin{tabular}{|l|c|c|c|c|}
\hline Sample & $\% \mathrm{C}$ & $\% \mathrm{O}$ & $\% \mathrm{~N}$ & $\% \mathrm{PA}$ \\
\hline Good Adhesion & 79.6 & 10.7 & 9.2 & 73 \\
Poor Adhesion & 83.7 & 9.0 & 7.2 & 57 \\
\hline
\end{tabular}

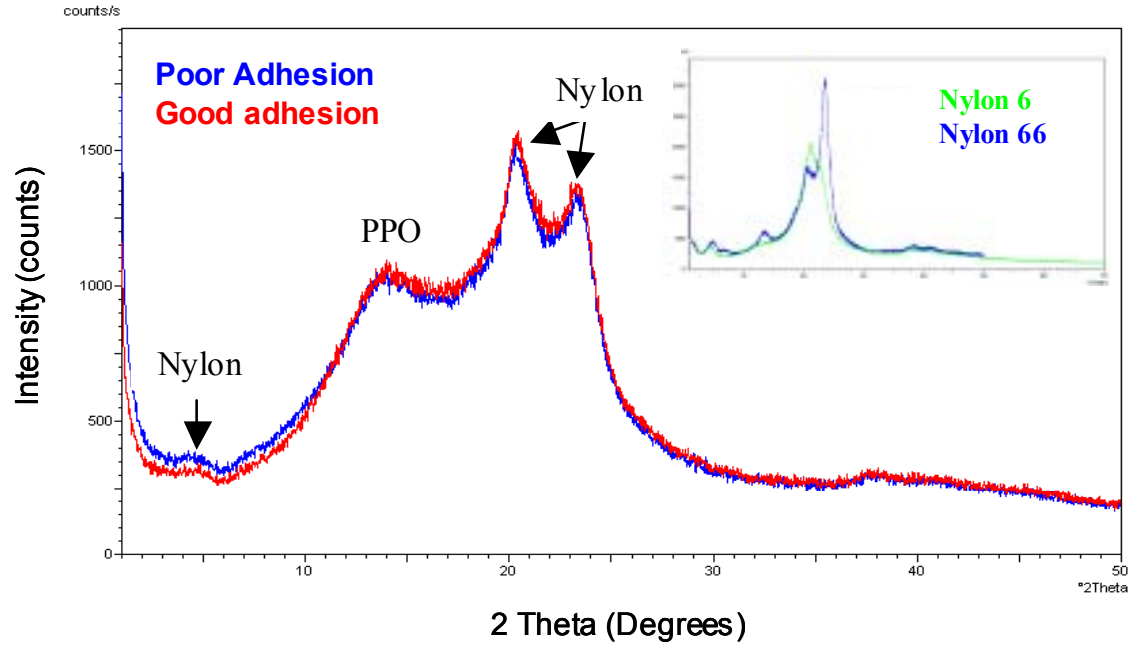

Fig. 2. Comparison of XRD spectra of samples with good and poor paint adhesion indicating comparable surface crystallinity. 